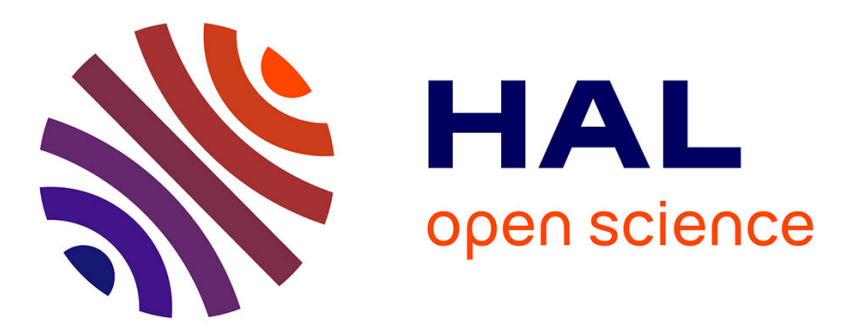

\title{
Application of the mol2vec Technology to Large-size Data Visualization and Analysis
}

Shojiro Shibayama, Gilles Marcou, Dragos Horvath, Igor Baskin, Kimito Funatsu, Alexandre Varnek

\section{- To cite this version:}

Shojiro Shibayama, Gilles Marcou, Dragos Horvath, Igor Baskin, Kimito Funatsu, et al.. Application of the mol2vec Technology to Large-size Data Visualization and Analysis. Molecular Informatics, 2020, 39 (6), pp.1900170. 10.1002/MINF.201900170 . hal-02950560

\section{HAL Id: hal-02950560 https://hal.science/hal-02950560}

Submitted on 13 Nov 2020

HAL is a multi-disciplinary open access archive for the deposit and dissemination of scientific research documents, whether they are published or not. The documents may come from teaching and research institutions in France or abroad, or from public or private research centers.
L'archive ouverte pluridisciplinaire HAL, est destinée au dépôt et à la diffusion de documents scientifiques de niveau recherche, publiés ou non, émanant des établissements d'enseignement et de recherche français ou étrangers, des laboratoires publics ou privés. 


\title{
Application of the mol2vec technology
}

\section{to large-size data visualization and analysis}

\author{
Shojiro SHIBAYAMA\$\#, Gilles Marcou\#, Dragos HORVATH", Igor I. Baskin+, Kimito FUNATSU§, Alexandre \\ VARNEK*\#
}

Dedication((optional))

\begin{abstract}
Generative Topographic Mapping (GTM) is an established dimensionality reduction method that simultaneously provides predictive modeling (by regression or classification) and data visualization. But large-size samples or large-size descriptors require a lot of resources to train a GTM. Therefore, it may be meaningful to apply some dimensionality reduction strategies prior to the training. This article proposes an artificial neural network learning technique called mol2vec to this aim. ISIDA Fragment descriptors and Morgan fingerprint are the herein used initial descriptor spaces.
\end{abstract}

This work specifically assesses whether "compression" to a few hundred dimensions of these initial descriptor spaces (typically, of dimensionality $>10^{3}$ ) by either the mol2vec procedure or, more classically, by Principal Component Analysis (PCA), may significantly speed up GTM training without compromising its predictive power in bioactivity classification tasks. This hypothesis has been confirmed by our results, furthermore showing that mol2vec generally outperformed PCA as dimensionality reduction method.

Keywords: Generative topographic mapping, QSAR, ISIDA descriptors, mol2vec, auto encoder

\section{Introduction}

Chemoinformatics approaches frequently use large numbers of molecular descriptors. Associated vector space dimensionality is large, while for specific QSAR modeling tasks, some descriptors may be redundant. Thus, it is common practice to use variable selection algorithms and dimensionality reduction techniques - with their inevitable loss of information and risks to introduce a bias.

The recent successes of deep learning in chemistry are tightly bound to the progress of text mining and the ability to deal with chemical graphs by analogy to texts. Deep learning is also a key issue in molecular description, for it has been shown that in the process of deep learning, the internal values stored on hidden neuron layers (latent variables) can be effectively used as molecular descriptors [1]. Since deep neural networks may "read" virtually any brute, non-preprocessed molecular representations (molecular graphs, SMILES strings canonicalized or not - and even $2 \mathrm{D}$ sketches in pixelized image formats) these latent variables can be considered as descriptors "invented" by the artificial intelligence. They should incapsulate (almost) all the information present in the molecular graph, albeit it will not be extracted following the chemical know-how used by an expert designing novel molecular descriptors. The latter would know, for example, to emphasize structural aspects that could, in principle, be inferred from the graph, but are difficult to extract. Typical examples are pharmacophore labels of atoms: an atom in a graph has its chemical context fully specified, and herewith its physico-chemical properties perfectly determined. Some are "obviously" hydrophobic, hydrogen bond acceptors or donors, while others participate in hard-to-predict proteolytic equilibria, which may render an unambiguous assignment of their charge and pharmacophore status difficult. An expert (or a dedicated in Silico model [2]) can help to clarify these ambiguities, and in so far might provide a competitive edge of expert-designed over deep-learned latent descriptors.

There is however a third way which does not prone an exclusive relying on either purely deep-learned or expertdesigned descriptors: namely, the usage of expert-based descriptors as primary molecular data, to be converted to lower-dimensionality "latent" terms by a deep learning procedure. This was introduced by Jaeger et al. [1] and Chakravarti [3], who used the word2vec [4] algorithm in order to propose a dense representation of the molecule called mol2vec.

We previously introduced an efficient chemical space mapping and modeling method: Generative Topographic

$\S$ Department of Chemical System Engineering, School of Engineering, University of Tokyo

7-3-1, Hongo, Bunkyo-ku, Tokyo, Japan

\# Department, Institution Laboratoire de Chemoinformatique, UMR7140 University of Strasbourg-CNRS

4, rue Blaise Pascal, 67000 Strasbourg, France *e-mail: varnek@unistra.fr, phone/fax: +33 (0)3 6885 1560

+ Faculty of Physics, Lomonosov Moscow State University, Leninskie Gory, 119991, Moscow, Russia

Supporting Information for this article is available on the WWW under www.molinf.com 
Maps (GTM) [5,6]. This tool is able to reduce the dimensionality of the chemical space to 2 dimensions in order to access to its informational content in analogy to geographic maps. The map is a probabilistic model of the data that can be used for QSAR modeling, database comparisons and many other applications [7-9]. However for high-dimensional initial spaces, the training algorithm uses a lot of CPU and computer memory. For this reason, molecular descriptors used for GTM are often processed by variable selection or dimensionality reduction techniques (using Principal Component Analysis, PCA).

PCA is the simplest - linear - dimensionality reduction method. The full set of principal components is equal to the effective rank of the molecular descriptor matrix, which can be lower than the actual number of molecular descriptors, if some of them are colinear or quasi-colinear It is common practice to keep high variance principal components, i.e. it is assumed that the information content of the ignored dimensions is low and irrelevant for the task on interest.

By contrast, mol2vec is a "distributed representation" using environment information of fragments in the molecular graph to compute lower-dimensional latent vectors. Based on the word2vec approach, assuming each word has various meanings depending on context, it regards molecular substructures as "words" in the context of neighboring fragments. Latent vectors are distancepreserving representation of the original molecules because similar fragments are encoded into similar latent vectors. The more the fragments are similar, the nearer the corresponding latent vectors are. The efficiency of mol2vec molecular descriptors has been demonstrated on several QSAR and QSPR studies [1,3].

In this work, we demonstrate that the mol2vec approach is efficient for chemical space modeling using the GTM approach, when compared to (a) on one hand, the "brute" descriptors (Morgan Fingerprints and ISIDA fragment counts), or (b) top PCA components of these brute descriptors (at same dimensionality as mol2vec terms). The quality of resulting maps was assessed following the "universal mapping" paradigm, advocating a chemical space mapping procedure able to demonstrate "polypharmacological competence" - i.e. able to support simultaneous prediction of many distinct biological properties based on a common GTM manifold [10]. The success of a dimensionality reduction approach in measured by the success of the GTM as a multi-task classification tool, that should be comparable to maps based on the brute descriptors. This article aims to show that such universal manifolds can be built much faster with mol2vec reduced descriptors of rather low dimensionality, without any significant loss in performance.

This paper is organized in Methods, Results and Discussion sections. The Methods section describes the technical details of the calculations. Then, Results and Discussion concerns the outcome of the realized benchmarking.

\section{Methods}

Herein we introduce descriptors, the concept of mol2vec and the map construction workflow. Details about the GTM methodology are described in the Appendix.

\subsection{ISIDA descriptors and Morgan fingerprint}

ISIDA descriptors are computed by our in-house software $[2,11]$, and represent integer vectors where each element is reporting the number of occurrences of the substructure (fragment) associated to it. Indexation of fragments is openended - the software creates a list of encountered fragments on the fly, while processing the compound set, and establishes the dictionary relating each vector element to its substructure. Once such dictionary is established, fragment counting in additional compounds needs to be constrained to it, in order to avoid reshuffling of fragment numbering (additional fragments, not encountered so far, will be added at the end of the dictionary, or simply ignored by user toggle). Thus, ISIDA Fragmentor does not presume a fixed set of substructures to be detected and counted but requires as input arguments a definition of a fragmentation strategy: rules defining how to break up the property-colored molecular graph. Atom "coloring" consists in replacing the default atomic symbol labels with precalculated relevant property labels such as pharmacophore type, force field type, and so on. In terms of fragment topology, one may opt between linear fragment, circular fragment, atom pair or atom triplet counts. Their acceptable (minimal and maximal) sizes of fragments to monitor are also user defined. In the following, ISIDA fragment descriptors were truncated: rare substructures were ignored to limit the total number of descriptors to around 9000 , even for the most "fine-grained" fragmentation schemes considered in this work. For a given molecule, each atom can eventually be described by a vector enumerating the molecular fragments it contributes to.

Morgan fingerprints (Morgan FP) [12], which is also closely related to ECFP [13], can be as well mimicked by ISIDA descriptors (albeit the original RDKit implementation is the one used here). Morgan FP monitors the presence/absence of circular fragments including all atoms within given radius (expressed in number of bonds) around a central atom. When the radius is set one, Morgan algorithm considers all fragments with atoms having at most one bond away from the central atom.

Usually, Morgan FP are hashed into a bit string, aiming to facilitate of fast bitwise similarity scoring, for which fixed-length vectors are desirable. However, this step is not performed in this study, because of bit collision risk. It is obvious that bit collision deteriorates predictive ability of QSAR models [14]. The fragments are used as they are recorded to feed the mol2vec encoder as explained below.

\subsection{Deep learning technique: $\mathrm{mol} 2 \mathrm{vec}$}

Mol2vec is an unsupervised learning approach that comprises a simple 3-layers linear dense neural network (Figure 1.). Mol2vec, originating from a method in natural language processing, reads a text file in which substructures of a molecule are sequentially listed, in order to learn a "distributed" representation. In this paper, we employed fastText [15] (version 0.1.0) and fasttext-python (version 0.8.3) as the mol2vec transformation tool. We did not use the original word2vec implementation, because fastText is reported to outperform the original word2vec. fastText is the improved 
version of word2vec, which considers sub-word information, like prefix and postfix. The input of the neural network for fastText is the sum of the sub-word count, while the output of the network is still one-hot representation of a fragment.

Each substructure is represented by a string, without spaces. The input vector for the neural network is the one-hot vector recording the presence of a fragment and the output is a onehot vector recording the presence of environing fragments in one line of a text file. Figure 1 exemplifies a situation where fragment $i$ is located next to fragment $j$. Mol2vec usually employs a skipgram algorithm in which a neural network attempts to predict the fragments (fragment $j$ ) surrounding the target fragment (fragment $i$ ) on a row. Two weight matrices are defining the network. The first one, $W$, encodes the highdimensional representation of an atom in the molecule into a small-dimensions latent vector. The second one, $W$, decodes the latent vectors into one-hot vector representation of the environment. In other words, the task that the neural network solves is multi-class classification, in which one fragment corresponds to one class. Before carrying out mol2vec, substructures must be listed in text format, in which every substructure is represented by its ID label (substructure SMARTS or SMILES or ISIDA fragment label). The details for the preparation of fragment text files is described in [1].

After the neural network's two weight matrices $W$ and $W$ are updated epoch by epoch, the first weight matrix $W$ is used for dimensionality reduction. The weight matrix $W$ is used to compute the dense vector. One molecule $n$ is represented by the sum of one-hot vectors of considered fragments, namely $\sum_{i=1}^{I} \boldsymbol{x}_{n, i}$ where $i$ denotes index of a fragment, I denotes the number of fragments in the molecule $n, \boldsymbol{x}_{n, i}$ denotes the one-hot encoding for the fragment. The dense vector $\boldsymbol{d}_{n}$ for molecule $n$ is expressed as follows.

$$
\boldsymbol{d}_{n}=W \sum_{i=1}^{I} \frac{\boldsymbol{x}_{n, i}}{\left|\sum_{i=1}^{I} \boldsymbol{x}_{n, i}\right|},
$$

where |. denotes the L1-norm. It is important that mol2vec also can be regarded as just a linear projection from the one-hot vector space to dense vector space, like PCA. The difference between mol2vec and PCA is how to learn the weight matrix $W$ (or loading matrix $P$ for $P C A$ ).

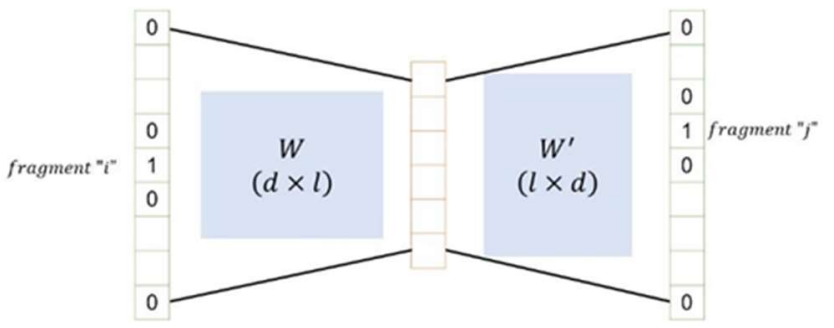

Figure 1. Schematic depiction of mol2vec. The input and output layers are one hot encoding of molecular fragments. The $W$ matrix maps the fragment "i" to a lower dimensionality latent space. The matrix $W$ decode the latent vector to fragment "j", which contributes to the environment of fragment " $i$ " in the molecule. The matrix size $d$ and / correspond to the number of fragments and the dimensionality of latent variables.

\subsection{Map construction procedure}

Structure-activity data for multiple targets was extracted and prepared in view of "Universal" map [16] construction. In this approach, 618 structure-biological activity sets were extracted from ChEBML 23 [17] and converted to binary (active/inactive) classification problems. Chemical structures from ChEMBL 23 were standardized before analysis as described in the previous "Universal" map article [16]. The resulting dataset contained 1,540,615 compounds with activities for 618 targets. A genetic algorithm (GA) was used to explore the hyperparameter space controlling the GTM algorithm setup. The key hyperparameter is the choice of the descriptor space to use - several descriptor files can be fed to the algorithm, which will try to select the ones that lead to better maps. However, here (vide infra), in order to generate the most proficient maps that can be supported by a given descriptor space, the algorithm was run having targeted descriptors as the only option, thus allowing it to focus on fitting the setup GTM parameters - grid size, number of RBF functions, etc. At given parameter choice, a manifold is built based on frame set compounds (random ChEMBL molecules, not related to the 618 structure-activity series, controlled by the frame set hyperparameter of the GA). Then, the manifold is used to solve 236 out of the 618 problems using a classification landscape method. We employed balanced accuracy (BA) as the classification performances. BA is the average sensitivity for each of the classes. For a $K$-class $(K=2)$ classification, BA is expressed as follows:

$$
B A=\frac{1}{K} \sum_{k=1}^{K} \frac{\#\{\text { correct prediction in class } k\}}{\#\{\text { samples in class } k\}},
$$

A 3-fold cross-validation repeated 4 times (with item reshuffling) of the 236 selection sets returns the corresponding 236 cross-validated BA scores. Their average penalized by their standard deviations <BA>$\sigma(B A)$ represents the fitness score of the map encoded by the current chromosome. The $\sigma$ above stands for the standard deviation over the selection sets. The maximization of the fitness score enables to select the best manifold in terms of stable performance of classification capability. Once the final maps are selected, the so-far not involved 382 external sets were also subjected to the cross-validated classification challenge. Therefore, the results section will explicitly report, for each considered map, both $\langle\mathrm{BA}> \pm \sigma(\mathrm{BA})$ over the 236 selection sets and the 382 external sets. Comparison of these means/standard deviations will highlight whether, and to what extent, selection sets are "privileged" because they contributed to select the maps.

In addition to classification proficiencies, the maps will be also characterized in terms of entropy and coverage. Relative information entropy is calculated as the information entropy divided by the possible maximum information entropy, which is the logarithm of the number of grid nodes on a GTM manifold. Map coverage (based on the entire $1.5 \mathrm{M}$ curated ChEMBL 23 compound set) is the ratio (number of grid nodes on which the cumulated responsibility $>1.0$ ) / (total number of nodes).

In this article, the studied molecular descriptors were Morgan Fingerprints of radius one (MFP1) and radius 2 (MFP2), and ISIDA Fragments, namely the 8 sets of descriptors shown to produce valuable Universal maps in the prior study [16] (Figure S13). For each of abovementioned initial descriptor spaces, the first 50,100, 150 and 200 principal components, and respectively 50,100 , 150 and 200-dimensional latent spaces produced by mol2vec models were challenged to serve as low- 
dimensional "alternatives" against their parent descriptor spaces.

For each of the MFP sets (MFP1 and MFP2) and their transformations as above-mentioned, a dedicated GA optimization for every descriptor space was run. The degree of freedom supporting descriptor space choice was fixed to the current option, while all other considered parameters were sampled normally. These include:

- frame set choice (like in the Universal map paper, multiple choices of random ChEMBL subsets were used),

- descriptor preprocessing/scaling choices (no transformation, $\mathrm{min} / \mathrm{max}$ rescaling, centering, centering and normalization to standard deviation, etc),

- number of grid nodes,

- number of RBF functions,

- regularization coefficient, and

- RBF width.

By contrast, with ISIDA descriptors, all the eight candidate spaces and their transformations were allowed to compete against each other within a single GA run.

\section{Results and discussion}

After benchmarking original molecular descriptors (Morgan FP and ISIDA), their PCA, and mol2vec descriptors in GTM-driven classification problems on multiple biological activities, we herein report their performances for classification tasks and their CPU cost. With Morgan FPs and dedicated GA-driven map-building for each descriptor space, (near-)optimal setups were discovered for each descriptor space, making the results comparable - i.e. informative about the intrinsic quality of that particular descriptor space. ISIDA descriptors and their transformations, however, are competing against each other in a single map-building GA. Some were selected to form the so-far best ISIDA-based maps, which does not preclude that other descriptor spaces might also serve to build robust maps. Not being selected may signal an intrinsic low quality but may also be due to insufficient sampling of this very vast problem space (premature focusing of the GA on the winning descriptors). Thus, the ISIDA descriptor results should not be interpreted as an actual benchmark, as only its positive results count: some transformed ISIDA descriptors were able to produce robust maps (of quality matching the universal maps specifically built [16] with unprocessed ISIDA descriptors) This validates the working hypothesis that mol2vec transformations of original ISIDA fragment counts is a valid strategy.

\subsection{Morgan fingerprints}

The brute Morgan fingerprint descriptors and their transformations lead to a total of 18 GTMs. For each GTM the Figures 2 and 3 report selection and external sets performances (upper), relative information entropy, and ChEMBL map coverage (lower graph).

Note that the upper graph "error bars" reflect the standard deviation of BA scores over the 236 "selection" and, respectively, 382 "external" targets. Some biological targets are intrinsically easier to predict than others. This may be due to intrinsically smoother SAR landscapes, or due to structurally biased training sets (homogeneous series of actives versus dissimilar decoys are notoriously easy to separate). Thus, the "error bars" should not be seen as an expression of randomness of quality scores. They are not an expression of some normally distributed source of noise (if they were, then all the differences would not have been statistically significant). The actual uncertainty of the mean BA scores should have been evaluated by repeatedly running the GA-driven map construction protocol and taking the standard deviation of the top solution of every run. This is unfortunately technically too expensive, and heavily dependent on the stochastic nature of evolutionary optimization, knowing that that cross-validated BA also depends on the random shuffling and splitting of the datasets into cross-validation folds. However, our previous experience with ISIDA-descriptorbased universal maps [16] showed that there is a tangible difference in performance between the top-ranked (at $B A=0.78 \pm 0.06)$ and bottom-ranked $(B A=0.76 \pm 0.07)$ ones. In Figure S13, performance of these "reference" Universal maps are plotted in the context of the herein obtained approaches. Therefore, a shift of $\mathbf{0 . 0 2}$ units of the BA average is, as far as our previous experience can tell, a significant difference in map quality.

MFP1 and MFP2 generated 17,270 and 544,331dimensional descriptors, respectively. In these figures, the left-most bars correspond to the unprocessed descriptors. It is expected that successful dimensionality reduction will minimize the loss of performance compared to these reference columns. This is the case for both PCA and mol2vec-compressed MFP1, at 50 and 100 dimensions in Figure 2. Paradoxically, the statistics drop for less aggressive dimensionality reduction at 150 and 200 dimensions. We therefore hypothesize that the number of effective dimensions is lower than 150. For PCA, this means that the linear algebra problem becomes ill defined and the larger number of principal components are numerically unstable. Thus at 150 dimensions, a substantial amount of numerical noise is introduced in the descriptors. The same holds for mol2vecprocessed terms. However, the total variance explained by the 200 first principal components culminates up to only $9 \%$ of the total variance (Figure 2.) for MFP1. This means that the essential information needed for classification problems is recovered very early in the few first principal components.

Figure 3 concerns MFP2. The number of initial dimension is so large that the GTM could not be produced using unprocessed descriptors: the CPU cost is prohibitive for that number of dimensions. Here, the mol2vec clearly outperforms the PCA approach. The performance of mol2vec for MFP2 in 50 dimensions outperformed the others, but performances of mol2vec for MFP2 in 100, 150, and 200 dimensions remain comparable to those obtained using MFP1. The chemical information content of the MFP1 and MFP2 datasets is the same. But it is diluted in a much larger number of dimensions in the latter case which represents $1.2 \%$ of the total variance with 200 principal components. Thus, the PCA problem becomes ill defined very early, resulting in a severe loss of information and each principal component contains much less information compared to their MFP1 analogues. The mol2vec approach is much more robust than ordinary fragment count descriptors. In the similar manner, a form of word2vec is superior to word counts in terms of accuracy in text classification tasks [18]. 


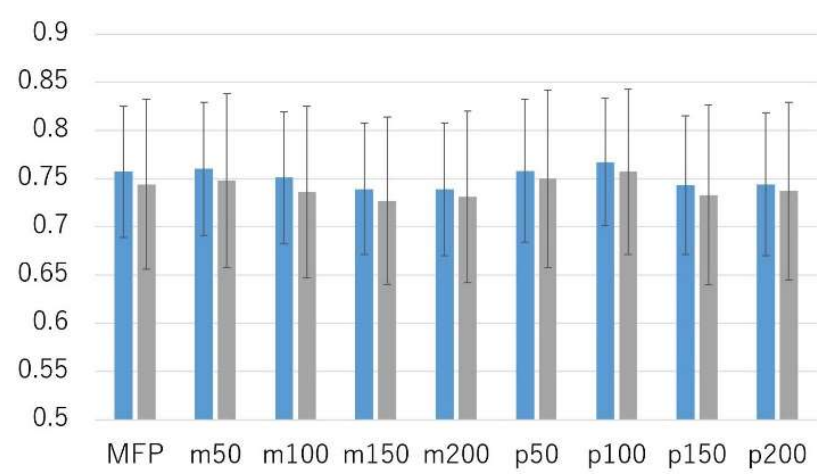

(a)

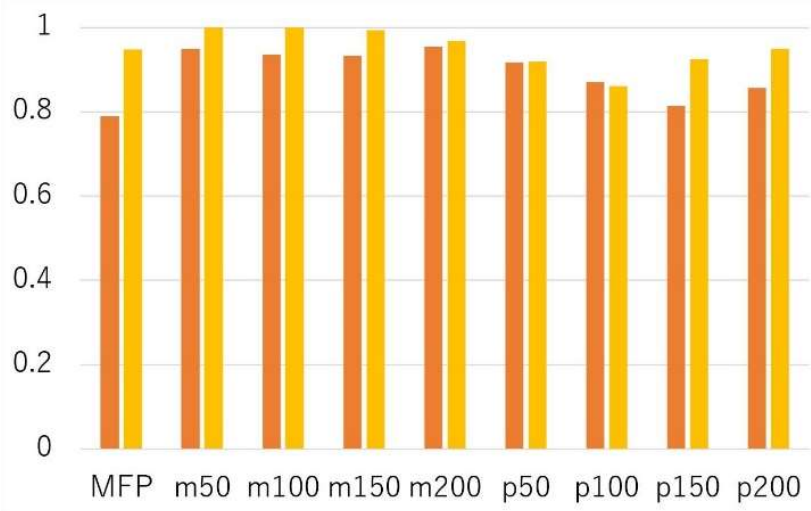

(b)

Figure 2. Results of descriptor sets for MFP1. (a) Balanced accuracies for selection sets (blue) and external sets (grey), (b) relative information entropy (orange) and map coverage (yellow). 'MFP', ' $m$ ' and ' $p$ ' in the labels indicate respectively Morgan fingerprint, mol2vec and PCA.

Note that MFP2 descriptors are clearly more "finegrained" than MFP1 - the number of more specific monitored fragments is larger, and the likelihood to see such a fragment shared by any two compounds decreases. Yet, if a fragment is not shared by a "critical mass" of molecules, its impact on activity cannot be determined in a statistically rigorous way, and they become "singleton" fragments, i.e. noise. Under these circumstances, chemical common sense would require to "cluster" several chemically related "singletons" into some generic fragment. For example, in a family of fragments containing a fluorine, chlorine, bromine or iodine atom respectively, each specific fragment may be not widespread enough for robust assessment of its impact on activity, but there might be enough data to do that for the generic halogen-containing fragment. Or, PCA does not "reason" in that way - it will rather regroup covariant fragments (which is not relevant in this case: the presence of the F-substituted fragment is by no means correlated with the $\mathrm{Cl}$-substituted one, etc). "Linguistic" analysis of fragment labels is more likely to regroup (Frag-F, Frag- $\mathrm{Cl}$, Frag-Br, Frag-I) into a generic "Frag-X". Based on the algorithms, it can be said that mol2vec learns which fragments are related, while PCA simply allocates similar scores for covariant fragments. PCA ignores totally the order of fragments and is not sensitive to rare fragments. We have so far no formal proof that this is the proper explanation for the outstanding mol2vec performance but the fact is that top performances for either Morgan Fingerprint-based Universal maps are achieved with the rather low-dimensional 50D mol2vec space b MFP2.

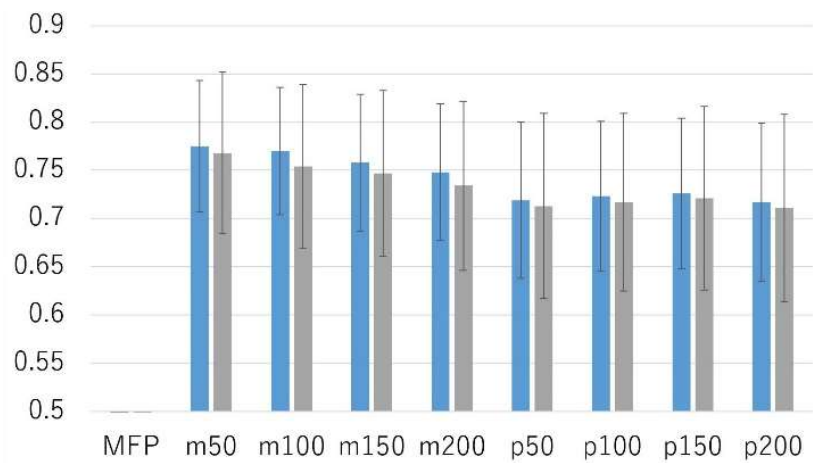

(a)

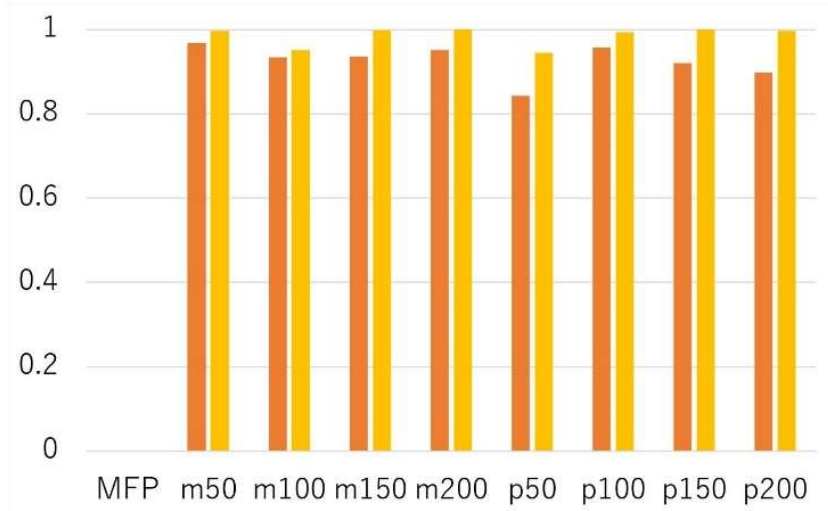

(b)

Figure 3. Results of descriptor sets for MFP2. (a) Balanced accuracies for selection sets (blue) and external sets (grey), (b) relative information entropy (orange) and map coverage (yellow). 'MFP', ' $m$ ' and ' $p$ ' in the labels indicate respectively Morgan fingerprint, mol2vec and PCA.

For comparison purposes, the experiment was repeated in adjusting the number of selected principal component for MFP1 and mol2vec dimensions to match the explained variance of the 50, 100, 150 and 200 MFP2. This resulted in 4, 7, 11 and 14 dimensions respectively. Results are collected in Figure 5.

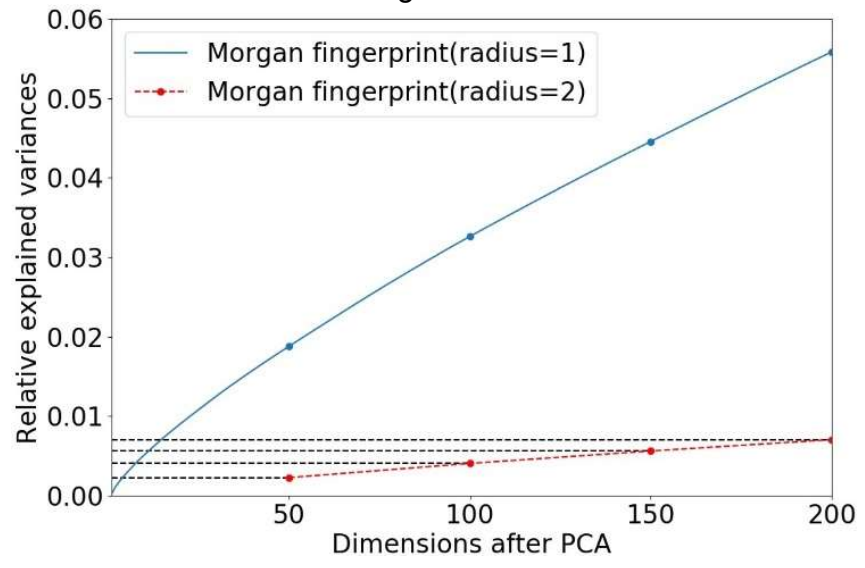

Figure 4. Cumulative sum of the fractions of explained variance by PCA at given number of principal components (on X) for both MFP 1 (blue) and MFP2 (red). Horizontal dashed lines started cross the MFP1 curve at dimensions that have almost the same relative explained variance. 
The variance explained by the first 200 principal components of Morgan FP 2 represents at most $1.2 \%$ of the total variance (Figure 2). Only $14 \mathrm{PCs}$ are required to explain that relative variance in MFP1 space, etc.

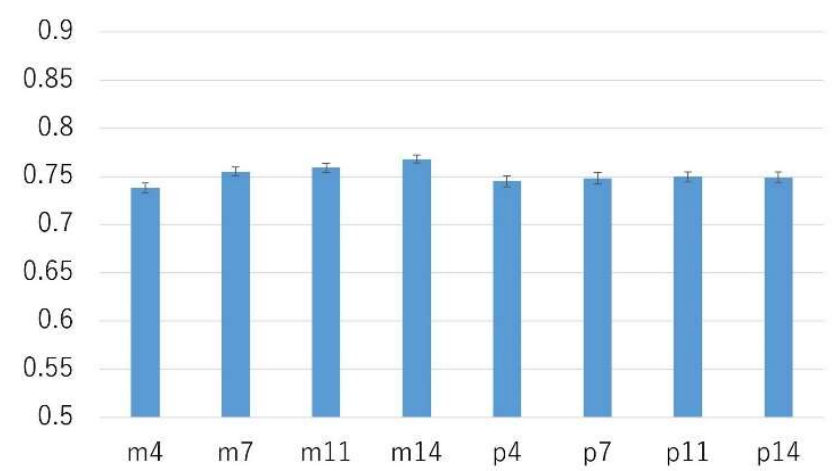

Figure 5. Performance of low-dimensional mol2vec and PCA descriptor sets of MFP1. Balanced accuracies for selection sets (blue) are reported on Y. ' $m$ ' and ' $p$ ' in the labels indicate respectively mol2vec and PCA.

Two observations emerge from Figure 5. First, the mol2vec descriptors outperform the PCA at 14 dimensions. Second, balanced accuracies for both mol2vec and PCA monotonously increase with the number of dimensions, within the range 4-14. Besides, the balanced accuracies were better than or almost equal to those for MFP 1 and 2 with 50, 100, 150, and 200 dimensions. This implies that the descriptor matrix is stable in terms of rank of a matrix within 4 to 14 dimensions. A matrix with $k$ rank only has at most $k$ orthogonal basis. PCA will provide unstable solution that may cause calculation errors, when the number of dimensions exceeds the rank of the matrix.

Fuzzy activity class landscapes based on some of the obtained GTMs are shown in Figures 6. and 7. (see supporting information for all activity landscapes). The brute MFP1, mol2vec, and PCA descriptors in 150 dimensions were used for Figure 6. (top left), Figure 6. (top right), and Figure 6. (bottom). The current illustrations concern target CHEMBL253 (the Cannabinoid CB2 receptor), where dark red zones are exclusively populated by CB2 (experimentally verified) non-binders, whilst dark blue zones exclusively harbor CB2-actives. Color transparency represents local population density. Intermediate colors correspond to zones where the two populations coexist (the discriminant power of these landscapes correspond to cross-validated $B A$ values of $0.75,0.77$ and 0.71 , respectively). Note that presence of large areas of intermediate color does not automatically translate into low BA values - unless those zones are also high-density areas. In this example, areas with "mingling" activity class assignments are on the overall sparsely populated, and BA values are significantly above the random selection levels. However, the stronger presence of yellow-green areas in the fuzzier PCA map is clearly marking a drop in discriminant power ( 0.71 versus $0.75 \ldots 0.77$ )

Mol2vec and PCA descriptors in 150 dimensions for MFP2 were used for the visualization, in the Figure 7. (left) and (right), respectively. Again, the PCA-based landscape is typically fuzzier and clearly less discriminant, as underlined both by its coloring and its rather low BA value. This figure is therefore illustrative of the tendency of principal component approach to filter out the details of the representation.

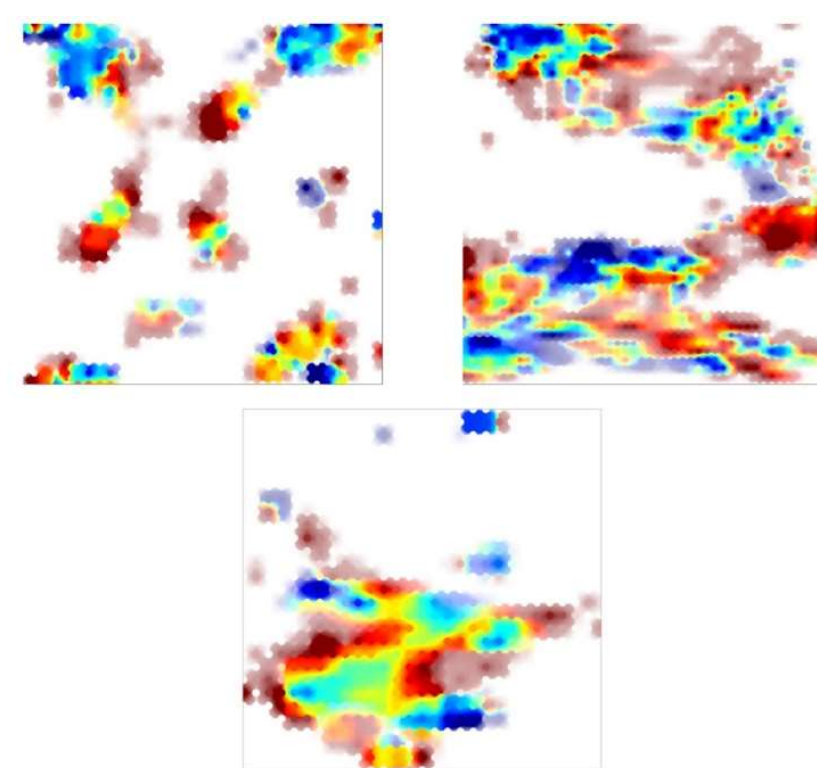

Figure 6. Activity against the target ChEMBL 253 landscaped on the GTMs based on MFP 1. (top left, $B A=0.75$ ) raw (top right, $\mathrm{BA}=0.77) \mathrm{mol} 2 \mathrm{vec}$ and (bottom, $\mathrm{BA}=0.71$ ) PCA (150 dimensions).

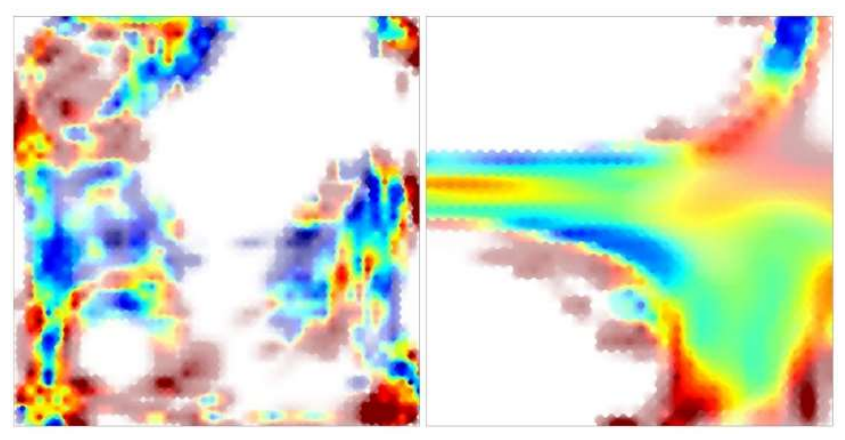

Figure 7. Activity against ChEMBL 253 landscaped on GTMs based on MFP 2. (left, BA=0.76) $\mathrm{mol} 2 \mathrm{vec}$ and (right, $B A=0.66$ ) PCA (150 dimensions).

It is not trivial to objectively compare computation times for GTM fitting, since each descriptor space required a map to be built at its optimal hyper-parameters. It is not straightforward to separate the time gains due to dimensionality reduction from the impact of GTM parameters on the convergence time of the algorithm. Note that even at common parameters, fulfillment of the convergence criterion may happen sooner or later, as a function of the descriptor space - thus, the time required to build a map in any descriptor space will invariably depend on training set and descriptors. As expected, there is no monotonic variation of computer time as a function of 50-100-150-200 kept dimensions in dimension-reduced spaces: map fitting typically took between 15 and 90 minutes. There are no outstanding differences between PCA and mol2vec-based spaces, albeit mol2vec based GTM are typically the longer ones to train. We hypothesize that this is due to the smoothing effect of filtering out low variance principal components. 
However, the key result here is that either PCA or mol2vec-reduced MFP1 descriptor spaces typically performed one order of magnitude faster than the full MFP1 fingerprint (while the full MFP2-based calculation never converged).

It must however be noted that, counterintuitively, the drastic dimensionality reduction will not lead to a decrease of descriptor file sizes - on the contrary, transformed descriptor files may occupy more than the double of disk space allotted to initial descriptor files. The reason behind this paradox is the sparse nature of Morgan and ISIDA fingerprints, for which only the populated fragments must be enumerated in .svm file (fragment ID:population level pairs are given only if the fragment of given ID is present at least once in the molecule). Albeit the total fragment number may be of several thousands, a descriptor line in a .svm file will feature typically no more than a few tens of integer (ID:level) pairs. The matrix of transformed descriptors is no longer sparse, and all the 50-200 columns must be explicitly written out as real numbers (still prefixed by the component number, in order to comply to input data format).

\subsection{ISIDA descriptors}

Results for ISIDA fragments descriptors are summarized in Figures S6-S12. (see Supplementary Information). The published ChEMBL23 universal maps [15] were based on 8 fragmentation types, based on precompiled fragment dictionaries characteristic for drug-like compounds.

As noted in Section 2.4, GA optimization visited a set of hyperparameters including descriptor types - allowing the various ISIDA fragment counts and their dimensionality-reduced derivatives to compete against each other for the realization of a top predictive GTM.

Results concerning PCA and mol2vec for truncated ISIDA descriptors in dimensions 50,100, 150, and 200 and as balanced accuracies for the universal maps are summarized in Figure S13. The results for dimensionality-reduced descriptors, whose balanced accuracies either outperformed or competed those of the corresponding ISIDA descriptors, were picked to show that several dimensionality-reduced descriptors were competitive in terms of classification capability. Universal maps were based on "winning" descriptor spaces out of a pool of various ISIDA fragmentation schemes and represent thus a good basis for expectation values of selection BA scores. In the current simulation, untransformed descriptor spaces also competed against their mol2vec and PCA transformations - thus, in light of the extremely stochastic nature of evolutionary algorithms, they might not have "rediscovered" or outperformed their original scores on universal maps. Failure to rank amongst the "winners" of such an evolutionary challenge may, but must not, be a sign that a given descriptor space is intrinsically flawed - it might simply not have been selected during the runtime. Meaningful are only positive results: if a descriptor space reaches or outperforms the to-date quality standards, there is no room to doubt its quality as a structure-activity modeling support. It is confirmed that some of mol2vec and PCA of ISIDA descriptors competed or even outperformed the original ISIDA descriptors. Conclusion

This study demonstrates that mol2vec technology is a promising method of dimensionality reduction, that can help to train accurate GTM with limited computer resources. Mol2vec preserves the multiple scales of the chemical space picture, while PCA tends to filter out the details. As a consequence, GTM with mol2vec generally outperformed GTM with PCA in terms of balanced accuracy.

To-date so-far best mean balanced accuracy scores of the multi-target classification problems addressed by the universal GTMs are well within range of results achieved by mol2vec (and, occasionally, PCA-based) reduced descriptors spaces. Apparently, information loss due to mol2vec transformations largely coincides with the information loss occurring at the intrinsic dimensionality reduction step provided by the GTM algorithm. Therefore, starting with a mol2vec-processed descriptor space with 100 dimensions or less does not incur any significant additional information loss than the one involved in projecting the $1000+$ dimensional initial spaces on a map. Mol2vec is particularly useful when the task involves hundreds of thousands of molecular descriptors, such as for the MFP2 case. In general, GTM based on mol2vec will facilitate classification/regression analyses with GTM allowing to improve GTM resolution. As a perspective, the behavior of these dimension-reduced descriptors in other machine-learning contexts should also be investigated.

\section{Conclusions}

This study demonstrates that mol2vec technology is a promising method of dimensionality reduction, that can help to train accurate GTM with limited computer resources. Mol2vec preserves the multiple scales of the chemical space picture, while PCA tends to filter out the details. As a consequence, GTM with mol2vec generally outperformed GTM with PCA in terms of balanced accuracy.

To-date so-far best mean balanced accuracy scores of the multi-target classification problems addressed by the universal GTMs are well within range of results achieved by mol2vec (and, occasionally, PCA-based) reduced descriptors spaces. Apparently, information loss due to mol2vec transformations largely coincides with the information loss occurring at the intrinsic dimensionality reduction step provided by the GTM algorithm. Therefore, starting with a mol2vec-processed descriptor space with 100 dimensions or less does not incur any significant additional information loss than the one involved in projecting the $1000+$ dimensional initial spaces on a map. Mol2vec is particularly useful when the task involves hundreds of thousands of molecular descriptors, such as for the MFP2 case. In general, GTM based on mol2vec will facilitate classification/regression analyses with GTM allowing to improve GTM resolution. As a perspective, the behavior of these dimension-reduced descriptors in other machine-learning contexts should also be investigated.

\section{Acknowledgements}

The authors thank Dr. Fanny BONACHERA for her technical support. The author S. S. thanks Japan's Ministry of Education, Culture, Sports, Science, and Technology (MEXT) and kind cooperative companies for financial support through Tobitate! Study Abroad Initiative and acknowledges financial support of Japan Society for the Promotion of Science (JSPS) in Grant- 
in-Aid for JSPS Fellows (DC2) program for the research stay in Strasbourg, France.

\section{Appendix}

\section{Appendix. 1 Generative topographic mapping}

Generative topographic mapping (GTM) is a generative form of self-organizing map. GTM basically fits its manifold onto samples in high dimensional space. GTM is fitted to data through maximization of log-likelihood. EM algorithm was used to maximize the log-likelihood, because GTM involves hidden variable $\boldsymbol{x}_{k}$, which is coordinate of nodes on a manifold.

First of all, a node of a GTM manifold $\boldsymbol{x}_{k}$ is projected into corresponding coordinate $\boldsymbol{y}_{k}$ in the data space as follows:

$$
\boldsymbol{y}_{k}=W \boldsymbol{\phi}\left(\boldsymbol{x}_{k}\right),
$$

where $W$ and $\boldsymbol{\varphi}$ indicate weight and output of radial basis functions (RBFs). $W$ forms a $d$-by-I matrix, where $d$ and $I$ denote dimensionality of the original space and the number of RBFs. Conditional probability of sample $n$ is expressed in the following:

$$
p\left(\boldsymbol{t}_{n} \mid \boldsymbol{x}_{k}, W, \beta\right)=\left(\frac{\beta}{2 \pi}\right)^{D / 2} \exp \left(-\frac{\beta}{2}\left|\boldsymbol{t}_{n}-\boldsymbol{y}_{k}\right|^{2}\right),
$$

where $\boldsymbol{t}_{n}, \beta$, and $D$ denote coordinates of sample $n$, inverse of variance, and the number of dimensions of $\boldsymbol{t}_{n}$. The probability of a node $\boldsymbol{x}_{k}$ for a sample $n$, which is called responsibility in the literatures, is expressed as follows by using Bayes' theorem:

$$
R_{k, n}=\frac{p\left(t_{n} \mid x_{k}, W, \beta\right)}{\sum_{k=1}^{K} p\left(t_{n} \mid x_{k}, W, \beta\right) p\left(x_{k}\right)} .
$$

This probability is called responsibility by the inventors. Then, GTM maximizes the log-likelihood of $\left(A^{*}\right)$ through EM algorithm. The log-likelihood is expressed in the following.

$$
L(W, \beta)=\sum_{n=1}^{N} \log \left(\frac{1}{K} \sum_{k=1}^{K} \exp \left\{-\frac{\beta}{2}\left|\boldsymbol{t}_{n}-y\left(\boldsymbol{x}_{k}, W\right)\right|^{2}\right\}\right)
$$

\section{Appendix. 2 Classification using GTM}

GTM is a visualization tool; however, by incorporating it into Bayes' theorem, GTM can be easily extended to a classification or prediction modeling method. In this part, the way of classification with GTM is presented.

The final goal of classification by GTM is to obtain posterior probability $p\left(c_{i} \mid \boldsymbol{t}_{n}\right)$ where $t_{n}$ and $c_{i}$ denote a sample in the original data space and class of $i$. The posterior probability of sample $n$ can be expressed as product of posterior probability of class $i$ at grid on a manifold $\mathrm{k} p\left(c_{i} \mid \boldsymbol{x}_{k}\right)$ and sample's probability distribution on manifold $p\left(\boldsymbol{x}_{k} \mid \boldsymbol{t}_{n}\right) . p\left(\boldsymbol{x}_{k} \mid \boldsymbol{t}_{n}\right)$ is equal to responsibility of sample $n$ at grid k, $R_{k, n} . p\left(c_{i} \mid \boldsymbol{x}_{k}\right)$ can be expressed by using Bayes' theorem, as follows:

$$
p\left(c_{i} \mid \boldsymbol{x}_{k}\right)=\frac{p\left(\boldsymbol{x}_{k} \mid c_{i}\right) p\left(c_{i}\right)}{\sum_{i} p\left(\boldsymbol{x}_{k} \mid c_{i}\right) p\left(c_{i}\right)} .
$$

Posterior probability $p\left(\boldsymbol{x}_{k} \mid c_{i}\right)$ is computed with responsibility as follows:

$$
p\left(\boldsymbol{x}_{k} \mid c_{i}\right)=\frac{1}{N_{i}} \sum_{n_{i}}^{N_{i}} R_{k, n_{i}} .
$$

$R_{k, n i}$ is responsibility for a node $k$ of sample $n_{i}$ as described in Appendix 1. In other words, the conditional probability of $\boldsymbol{x}_{k}$ for class $i$ is the average of responsibility for samples of class $i$ in a training dataset. The sample $n_{i}$ belongs to class $i$.

$$
p\left(c_{i} \mid \boldsymbol{t}_{n}\right)=\sum_{k=1}^{K} p\left(c_{i} \mid \boldsymbol{x}_{k}\right) R_{k, n} .
$$

The posterior probability of class $i$ for any sample $n$ can be computed, because responsibility $R_{k, n}$ for a new sample can be computed using the optimized weight matrix $W$ and $\beta$.

\section{References}

[1] S. Jaeger, S. Fulle, S. Turk, J. Chem. Inf. Model. 2018, 58, 27-35.

[2] F. Ruggiu, G. Marcou, A. Varnek, D. Horvath, Mol. Inf. 2010, 29, 855-868

[3] S.K. Chakravarti, ACS Omega. 2018, 3, 2825-2836.

[4] T. Mikolov, I. Sutskever, K. Chen, G. Corrado, J. Dean, Proc. NIPS. 2013.

[5] H.A. Gaspar, G. Marcou, D. Horvath, A. Arault, S. Lozano, P. Vayer, A. Varnek, J. Chem. Inf. Model. 2013, 53, 12, 33183325.

[6] C.M. Bishop, M. Svensén, C.K.I. Williams, The Generative Topographic Mapping, Neural Comput. 1998, 10, 215-234.

[7] N. Kireeva, I.I. Baskin, H.A. Gaspar, D. Horvath, G. Marcou, A. Varnek, Mol. Inf. 2012, 31, 301-312.

[8] S. Takeda, H. Kaneko, K. Funatsu, J. Chem. Inf. Model. 2016, 56, 1885-1893.

[9] K. Mishima, H. Kaneko, K. Funatsu, Mol. Inf. 2014, 33, 779789.

[10] P. Sidorov, H. Gaspar, G. Marcou, A. Varnek, D. Horvath, J Comput Aided Mol Des. 2015, 29, 1087-1108.

[11] M. Glavatskikh, T. Madzhidov, V. Solov'ev, G. Marcou, D. Horvath, J. Graton, J.Y. Le Questel, A. Varnek, Mol. Inform. 2016, 35, 70-80.

[12] H.L. Morgan, J. Chem. Doc. 1965, 5, 107-113.

[13] D. Rogers, M. Hahn, Extended-Connectivity Fingerprints, J. Chem. Inf. Model. 2010, 50, 742-754.

[14] A. Kensert, J. Alvarsson, U. Norinder, O. Spjuth, Evaluating parameters for ligand-based modeling with random forest on sparse data sets, J. Cheminform. 2018, 10, 49

[15] P. Bojanowski, E. Grave, A. Joulin, T. Mikolov, arXiv preprint arXiv:1607.04606, 2016.

[16] I. Casciuc, Y. Zabolotna, D. Horvath, G. Marcou, J. Bajorath, A. Varnek, J. Chem. Inf. Model. 2018, 59, 1, 564-572.

[17] A. Gaulton, L.J. Bellis, A.P. Bento, J. Chambers, M. Davies, A. Hersey, Y. Light, S. McGlinchey, D. Michalovich, B. AlLazikani, J.P. Overington, Nucleic Acids Res. 2012, 40, $1100-1107$

[18] A. Joulin, E. Grave, P. Bojanowski, T. Mikolov, Bag of Tricks for Efficient Text Classification, arXiv preprint. arXiv:1607.01759. 2016.

Received: ((will be filled in by the editorial staff)) Accepted: ((will be filled in by the editorial staff)) Published online: ((will be filled in by the editorial staff) 
